\section{Dropped head syndrome due to neuromuscular disorders: Clinical manifestation and evaluation}

\author{
Ahmet Z. Burakgazi, Perry K. \\ Richardson, Mohammad Abu-Rub \\ Virginia Tech Carilion School of \\ Medicine, Roanoke, VA, USA
}

inflammatory polyneuropathy (CIDP), ${ }^{11}$ neuromuscular causes include myasthenia gravis (MG), ${ }^{12-14}$ Lambert-Eaton myasthenia syndrome (LEMS), ${ }^{15}$ muscular causes includes primary inflammatory such as polymyositis, ${ }^{16}$ scleromyositis, ${ }^{17,18}$ isolated inflammatory axial myopathy, ${ }^{19}$ primary non-inflammatory such as nemaline myopathy, mitochondrial myopathy, ${ }^{20-22}$ congenital myopathy, ${ }^{23}$ FSHD, ${ }^{24}$ and isolated neck extensor myopathy (INEM). ${ }^{19}$

\section{General approach: clinical mani- festation and evaluation}

DHS occurs as a result of weakness of posterior neck muscles. It usually disappears with supine position. The common chief complaints are "chin on the chest" and "difficulty maintaining a forward gaze". It may contribute to dysphagia and has cosmetic and social implications.1,12,19,25 Semispinalis cervicis and semispinalis capitis are primarily responsible for neck extension. DHS is thought to be directly related to weakness or laxity of the semispinalis cervicis muscles. 1,25

During the evaluation of dropped head, providers first should rule out structural diseases of the cervical spine. To differentiate NM causes from non-NM causes is very important. In NM causes, a significant weakness of neck extensors is usually observed. In non-NM causes, imbalance of neck muscle tone leads to dystonic anterocollis. It is paramount important to identify potential treatable causes. $1,2,12,19,25$

A deliberate and detailed history taking is a key factor for an accurate diagnosis and important clues can be gathered from the history. Firstly, a provider should identify pattern of involvement and characteristics of weakness. These questions should be asked if a patient has isolated neck extensor weakness or diffuse weakness; if a patient has fatigable weakness or diurnal variation; if there is any cranial and/or bulbar muscle involvement; if there is any respiratory muscle weakness; and if there are any associated symptoms such as autonomic symptoms. Detailed family and developmental history should be taken as well as history of onabotulinumtoxin A injections. 1,2,16,19,25,26

After detailed history, cautious and complete physical examination should be performed to collect more clues for an accurate diagnosis. In the physical exam, upper and/or lower motor signs, muscle wasting/atrophy (as in motor neuron disease), skin findings (as in dermatomyositis), ocular and bulbar weakness (as in MG), facial weakness (as in FSHD), highly selec-
Correspondence: Ahmet Z. Burakgazi, Neuroscience Section, Department of Medicine, Virginia Tech Carilion School of Medicine, 3 Riverside Circle, Roanoke, VA 24016, USA.

Tel.: +1.540-521-4592.

E-mail: drburakgazi@yahoo.com

Key words: Dropped head syndrome, neuromuscular disease.

Contributions: the authors contributed equally.

Conflict of interest: the authors declare no potential conflict of interest.

Funding: none.

Received for publication: 11 June 2019 Accepted for publication: 18 June 2019.

This work is licensed under a Creative Commons Attribution NonCommercial 4.0 License (CC BY-NC 4.0).

(C) Copyright: the Author(s), 2019

Licensee PAGEPress, Italy

Neurology International 2019; 11:8198

doi:10.4081/ni.2019.8198

tive weakness pattern (as in IBM), and pelvic girdle weakness (as in most generalized myopathies) should be assesses carefully. $1,2,25,27$

Following physical examination, a provider should decide if further imaging tests are warranted. X-rays can be ordered to rule out kyphosis, fractures, and arthritis. Magnetic resonance imaging (MRI) is superior to Computed tomography (CT) to imagine soft tissue includes tendons, ligaments, fascia, fibrous tissues, fat, synovial membranes, muscles, and nerves. ${ }^{28,29}$ Contrast enhancement is sensitive to detect inflammation. MRI imaging demonstrates fatty infiltration, edema, and/or atrophy of the neck musculature. ${ }^{28}$ Radiological imaging particularly MRI is useful for determining biopsy sites in selective cases. ${ }^{28,29}$

Electrodiagnostic (EDx) studies provide valuable information in the diagnosis. 30,31 The needle study should include cervical and thoracic paraspinal, sternocleidomastoid muscle, and trapezius muscles. Facial and tongue muscles should be tested if any weakness is detected. Electromyographer should be careful not to overestimate paraspinal muscles finding during the needle testing since normal paraspinal motor unit action potentials have smaller amplitudes and a shorter duration, but the recruitment is normal as opposed to reduced or early recruitment. ${ }^{30-32}$

Muscle biopsy can be considered in 
selected cases. Most of the biopsies are taken from shoulder girdle muscles including deltoid and trapezius muscles. 30,33 Biopsy from cervical paraspinal muscles are more valuable, but more challenging because small and larger muscles are intermingled and it is difficult to know exactly which muscles should be biopsied. ${ }^{30,32}$ Muscle biopsy may provide valuable information particularly in diagnosis of inflammatory muscle diseases such as size variability with fibrosis (Figure 1) and inflammation with some necrotic fibers (Figure 2). Ultrasound guided biopsy may be helpful, but not is not widely used.

\section{DHS in amyotrophic lateral sclerosis}

Dropped head is a rare manifestation of ALS occurring around $1-3 \%$ of patients. ${ }^{34}$ Dropped head is usually an early feature of ALS but it is usually not the presenting symptom. Neck flexor muscles weakness is usually worse than neck extensor muscles in ALS patients. DHS in ALS patients has been reported in few case reports. 2,3,34 A single-hospital-based retrospective study 34 from Japan including 105 sporadic ALS patients showed the incidence rate of DHS was $2.9 \%(3 / 105)$. The ages were 56,63 , and 53 years. The initial symptoms of the patients were bulbar palsy in two patients and weakness of the upper limb in one patient. ${ }^{34}$ The onset to DHS was 14,4 and 26 months after the onset of illness. Patients with prominent legs weakness did not develop DHS in their study. 34
Another retrospective study, 3 including 683 patients with ALS from India showed 9 of the patients $(1.3 \%)$ had severe weakness of neck extensor muscles observed as an early feature, or developing during the later stages of the disease. The mean of affected patients was $53.3( \pm 10.3)$ (range 33 to 65$)$. Six of them developed head drop as an early feature (mean interval from onset of illness was 11.6 months) and three has late onset (between three and eight years after onset). ${ }^{3}$ In their series, none of patients developed respiratory difficulties but all had swallowing difficulties and social embracement. ${ }^{3}$
The pathogenesis of DHS in ALS may be preferential involvement of paraspinal anterior horn cells. Electromyography (EMG) test shows denervation with no myopathic features. Muscle biopsy is usually consistent with neurogenic atrophy.2,12

DHS may cause neck pain and discomfort in ALS patients. DHS can cause severe impairments in activities of daily living (ADL) such as eating, dressing so ALS patients with DHS are more dependent in ADL. ${ }^{12,34}$ We advise ALS patients with DHS to wear a neck collar, which improve their posture, swallowing, and neck pain.

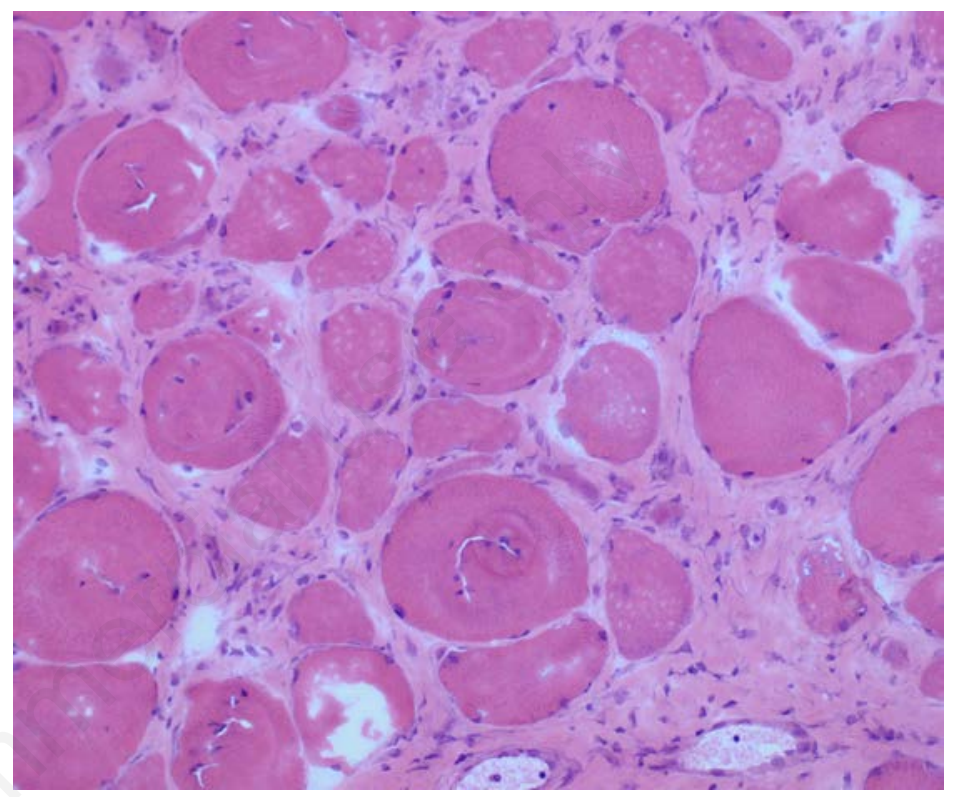

Figure 1 . The picture demonstrates size variability with fibrosis.
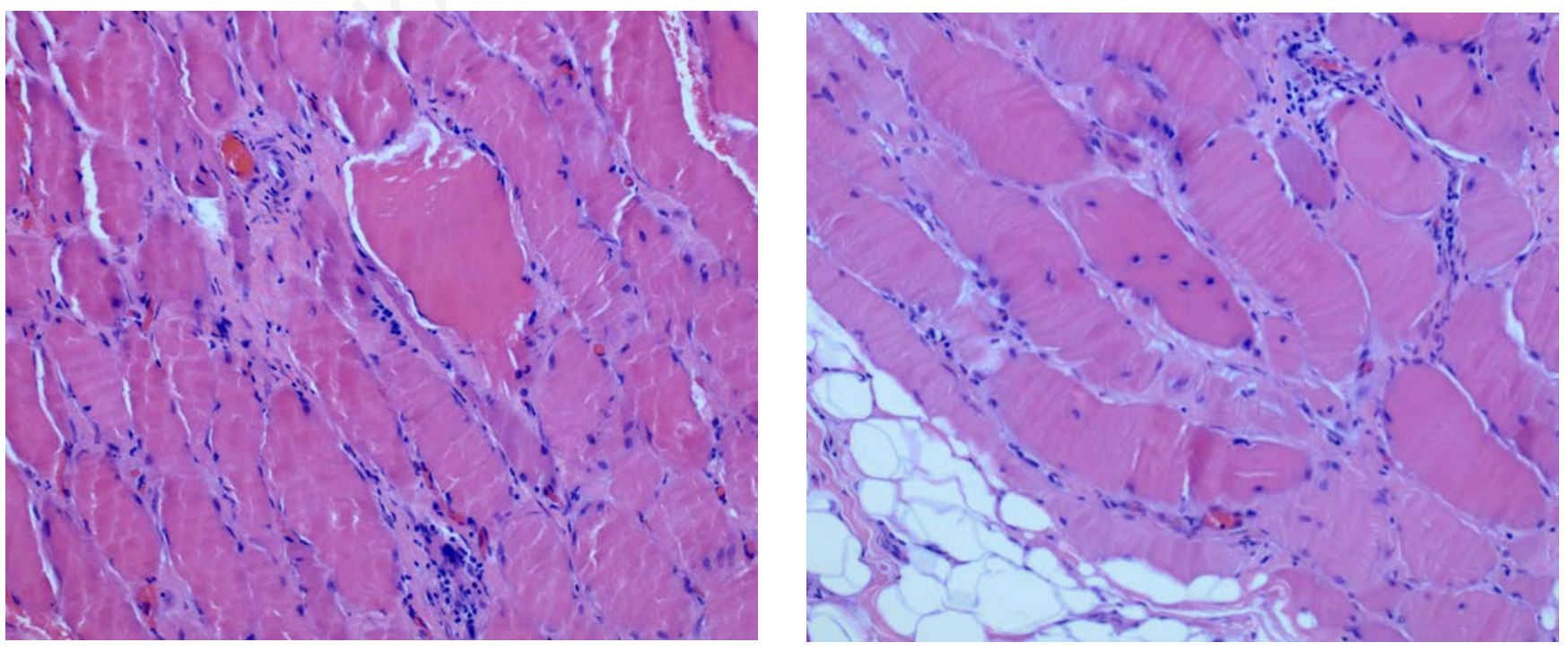

Figure 2. The pictures demonstrate inflammation with some necrotic fibers. 


\section{DHS in neuromuscular junction disorders}

\section{Myasthenia gravis}

Drooped head is a well-recognized feature of MG even in the absence of ocular or bulbar signs. It can be associated with or contribute to respiratory muscle weakness. Dropped head can be the presenting sign of only sign of MG. One retrospective study, 35 including 146 patients with generalized MG showed 15 patients $(10 \%)$ of the patients developed head drop. Of these 15 patients, acetylcholine receptor binding antibodies (AchR Ab) were positive in 14, and antiMuSK antibodies positive in 1.35 Dropped head symptoms in MG patients with positive AchR Ab improved with treatments, but the patient with positive anti-MuSK Ab was refractory to multi treatments including prednisone, plasmapheresis, and mycophenolate mofetil. Shortcoming of the study was no imaging, EMG or muscle biopsies findings were reported. ${ }^{35}$

Another case series study reported 13 MG cases with dropped head. Of those patients, 8 were positive with AchR Ab; 3 were positive with MuSK Ab; and 2 were sero negative. Eight of the patients had cervical imaging and one showed atrophic muscle changes. Two of them underwent thymectomy. Six patients had full remission, and seven has significant improvement with therapy. The outcome is usually good with effective control of myasthenic symptoms. Paraspinal muscles biopsies were not performed in the study.

Treatment-refractory MG cases should be reassessed for possible concomitant axial myopathy. First case was reported by Rodolico in 2014.36 The patient presented with ocular, bulbar, and shoulder girdle weakness preceded head drop. The initial blood work up showed normal CK, positive AchR binding antibodies. Cervical MRI showed atrophy and fatty replacement of neck extensor muscle. EMG test showed sporadic fibrillation and myopathic motor unit action potentials (MUAPs) limited to paraspinal muscles. Deltoid muscle biopsy showed scattered centralized nuclei. The case was refractory to pyridostigmine and/or prednisone. ${ }^{36}$ Eight more cases with MG and concomitant axial myopathy have been reported since then. Seven with positive AchR Ab and positive MuSK Ab. All patients' EMG showed myopathic MUAPs in paraspinal muscles. Three had biopsies showing fiber atrophy, endomysial inflammation with fibrosis. Five were refractory to multi-therapy including thymectomy, pyridostigmine, prednisone, intravenous immunoglobulins (IVIG), plasmapheresis, and mycophenolate mofetil. Only one had full remission and two showed some improvements.

The pathological relationship between MG and axial myopathy is unclear. Most of the reported cases are in their late $70 \mathrm{~s}$ or early $80 \mathrm{~s}$. Mechanical stretch injury, loss of tissue elasticity with aging, chronic use of steroids/immunosuppressive agents may contribute myopathy process. ${ }^{36-38}$ EMG test can be helpful for differential diagnosis but should be performed by experienced electromyographer to avoid under or overestimate. Cervical MRI imaging is useful to detect atrophy of cervical paraspinal and surroundings muscles. ${ }^{36-38}$

In our opinion, cervical paraspinal muscle biopsy and cervical imaging are not required as a part of the initial work up in myasthenia patients with head drop since it will not change treatment approaches. Cervical paraspinal muscle biopsy and cervical imaging should be considered in treatment-refractory cases.

\section{Lambert-Eaton myasthenic syndrome}

LEMS is an autoimmune disease affecting neuromuscular junction due to antibodies production against voltage gated calcium channels (VGCC). DHS is very rare clinical presentation of LEMS. LEMS syndrome presenting with DHS has been reported twice. The first case was associated with antibodies against P/Q VGCC and his symptoms and electrophysiological abnormalities improved with oral prednisone following plasmapheresis. ${ }^{15}$ The second case was associated with autoantibodies against N-type VGCC. ${ }^{39}$ The patient presented with symmetric paresis $(3 / 5)$ of the head extensors with pronounced muscle atrophy. She had more pronounced weakness in the evening. She had partial but sudden restoration of weakness with intravenous edrophonium. The initial blood test was negative for myasthenia gravis antibodies. She was initially admitted for sero-negative MG and treated with pyridostigmine and methyl-prednisolone. She was discharged home in an improved state. She was readmitted after 4 weeks with worsening of neck weakness and a progressive general muscle weakness as well as dysarthria and dysphagia. 39 The further work up showed positive N-type VGCC and was treated with unmodified medication with continuous and significant improvement. The serum titer of N-type VCGG specific antibodies dropped to normal that correlated with clinical improvements. 39

\section{DHS in peripheral neuropathy}

\section{Chronic inflammatory demyelinat- ing polyneuropathy}

DHS is very rare presentation of CIDP. Two cases have been reported on DHS with CIDP. In the first case report, a 19-year-old female with history of Guillain-Barre syndrome with fair recovery developed marked weakness of the neck extensors that she was unable to light her head. The detailed worked-up including EDx testing, cerebral spinal fluid (CSF) analyses were consistent with CIDP. She was treated with plasmapheresis, prednisone, and azathioprine but she did not have any remarkable improvement in neck extensors and shoulder girdle muscles. She had to use cervical collar for neck stabilization. ${ }^{11}$ The second report was reported in 2006,40 a 63 year-old patient presented with slowly progressive weakness of the neck extensor muscles developing a dropped head syndrome over a 12 months period. The detailed investigation including EMG, MRI, CSF analysis were inconclusive. Within two years, she developed fasciculations, atrophic paresis of the limbs and respiratory muscle involvement with consecutive respiratory failure. The postmortem neuropathological assessment demonstrated CIDP with involvement of the autonomic ganglia and the intramural plexus. Due to the complexity of the case the clinical diagnosis of a CIDP as cause of the dropped head syndrome was able to make after the postmortem pathological examination. 40

\section{Charcot Marie tooth disease type 4C}

In another interesting report, an 11year-old boy presented with proximal muscle weakness with facial involvement as well as dropped head and severe scoliosis. The detailed work up showed sensorimotor demyelinating polyneuropathy and genetic test confirming Charcot Marie Tooth disease type 4C. ${ }^{41}$ Therefore early-onset inherited polyneuropathies should also be included in the differential diagnosis of patients with proximal muscle wasting associated with dropped head syndrome. 41

\section{DHS in muscle disorders}

The DHS occurs in a variety of muscular disorders including primary inflammatory such as PM, 16,42 scleromyositis (SM), ${ }^{17,18}$ isolated inflammatory axial myopathy ${ }^{19}$, primary non-inflammatory nemaline myopathy, mitochondrial myopathy, ${ }^{20-22}$ congenital myopathy, ${ }^{23}$ FSHD, ${ }^{24}$ and isolated neck extensor myopathy. ${ }^{19}$ 
Wide spectrum pathological processes associated with the myopathic DHS ranging from non-inflammatory muscle necrosis to a classic inflammatory myositis.

\section{Polymyositis/Scleromyositis}

Inflammatory myopathies can cause DHS. In one report, two patients with myopathic dropped head syndrome presented with a predominant weakness of the neck extensor muscles. One patient had a good response to steroid and a histopathological assessment revealed PM.16 The other one did not have a good response to steroid but the weakness was stabilized after initial progression. The muscle biopsy was consistent with a necrotizing myopathy with no evidence of inflammation. ${ }^{16}$ In another report, ${ }^{42}$ a 69 year-old male with history of PM presents with DHS due to a relapse of DM. DHS improved by $60-70 \%$ with 2 months with methyl-prednisone $(80 \mathrm{mg} / \mathrm{d})$ that was tapered down to $4 \mathrm{mg}$ within 8 months. 42

Two patients were reported with DHS associated with SM, also known as scleroderma-PM overlap syndrome. ${ }^{17}$ One of the cases,53 year-old women, presented with DHS with mild dysphagia. ${ }^{17}$ She had wasting of the neck extensor as well as the shoulder girdle muscles. Serum creatinine kinase, lactate dehydrogenase, aspartate and alanine aminotransferase were elevated. EMG findings showed myopathic process with early recruitment in deltoid, iliopsoas, and quadriceps muscles. A muscle biopsy of the left deltoid showed a mild and diffuse process of necrosis and regeneration associated with major perivascular inflammatory infiltrate locates in endomysial, perimysial and epimysial tissues. The autoantibody screen revealed positive antinuclear antibody (1/640), mildly positive antineutrophilic cytoplasmic antibody (1/40), positive rheumatoid factor at $128 \mathrm{UI} / 1$ (normal b30), and positive anti PM-Scl antibody. Cervical MRI without gadolinium was unremarkable. She was treated with prednisone $1 \mathrm{mg} / \mathrm{kg}$. The dysphagia resolved within 2 weeks and motor weakness rapidly improved. Within two months, she was able to keep her head straight. Her condition progressively improved for one year, then remained stable.17 The other case, 18 51year-old man presented with neck pain and inability to maintain the head erect. The detailed blood, pathological investigations were consistent with scleromyositis. He was treated with prednisone on a $60 \mathrm{mg} / \mathrm{d}$ stepdown dosage with subcutaneous methotrexate at a dose of $25 \mathrm{mg} / \mathrm{wk}$, which resulted in remarkable improvement in neck weakness and limitations within 1 month with normalized muscle enzymes. ${ }^{18}$

\section{Isolated neck extensor myopathy}

INEM was first described in four patients in 1996.19 The author reported four cases presented with neck extensor weakness. The age ranged from 65 to 85 years old. Only one patient had mild flexion, and shoulder abduction weakness. EMG showed myopathic findings in cervicothoracic paraspinal muscles. Results of cervical paraspinal muscle biopsies demonstrated nonspecific myopathic features. The detailed neurological investigations were inconclusive. 19 Treatment trials with pyridostigmine bromide and prednisone were attempted in 3 of them with no clinical improvement. All patients had initial progression of weakness, followed by stabilization of neck weakness with no changes in follow up. ${ }^{19}$ The responsiveness of treatment of INEM is debatable. INEM usually has a poor response to immunosuppressive therapy because it is a non-inflammatory myopathy. ${ }^{19}$ However, recent publications have challenged this general acceptance. ${ }^{43-}$ 45 Muppidi et al. 43 reviewed four patients with INEM who received one or more immunosuppressive agents and showed neck strength improved by one or greater on the Medical Research Council scale in all patients with a peak response between 3 and 6 months after treatment initiation. They recommended a trial of immunosuppressive agents to patients with INEM. ${ }^{43}$ As a definition, INEM is a non-inflammatory myopathy but focal myositis could be missed on muscles biopsy that may explain immunosuppressive responsiveness in some INEM cases. 43,45

\section{Facioscapulohumeral muscular dys- trophy}

FSHD is rare reason of DHS. It was reported that a patient with FSHD developed dropped head caused by severe wasting of neck muscles. 46 In Kazakov et al. study, 47 the review of 200 FSHD patients showed only 4 patients had slight or moderate weakness of neck extensor muscles. In the same series, some patients with severe muscle atrophy and weakness of muscles fixing the scapula demonstrated visible dropped head because of abnormal posture of the shoulder girdle. ${ }^{47}$

\section{Anti-glutamic acid decarboxylase- associated inflammatory myopathy}

DHS is a complex and perplexing condition that has been reported in different neurological disease. In one of the interesting report, ${ }^{48}$ a 78 -year-old woman presented with a 10-month history of difficulty in keeping her head up during sitting and standing positions. The detailed investigation ended up with a diagnosis of anti-glutamic acid decarboxylase-associated inflammatory myopathy. Her symptom improved after intravenous immunoglobulins $(0.4 \mathrm{~g} / \mathrm{kg} /$ day for 5 days $)$ followed by prednisone $25 \mathrm{mg}$ /day for 2 months. ${ }^{48}$

\section{Adult-onset nemaline myopathy}

Another cause of dropped head is adultonset nemaline myopathy. 49 In one case, ${ }^{49}$ reported in 1999, a 59-year-old Greek painter complained of progressive pain and weakness in neck extensors, proximal legs, and shoulders for 1 year. Cramps, twitching, and atrophy were detected in the affected muscles. EMG showed myopathic abnormalities in proximal muscles with normal nerve conduction studies (NCS). Muscle biopsy demonstrated prominent muscle fiber atrophy and centrally located nuclei and electromicroscopy revealed that the atrophic fibers contained large arrays of osmiophilic oval structures, composed of Zband material. ${ }^{49}$ In the same report, the authors reviewed previously published 11 well-documented cases of adult-onset nemaline myopathy and they showed that neck weakness was mentioned in seven cases, and four of them had severe extensor weakness. ${ }^{49}$ After that report, several cases of nemaline myopathy related DHS are reported. ${ }^{21,22,50,51}$

\section{Amyloidosis}

In another report, a 77-year-old man with history of lymphoma and neck radiation presented with neck extensor weakness. ${ }^{52}$ Needle EMG showed myopathic changes, and biopsy of cervical paraspinal muscle demonstrated amyloid deposition in blood vessels. ${ }^{52}$ In another similar case, the further investigation revealed DHS with proximal myopathy caused by AL amyloidosis. ${ }^{53}$ Thus amyloidosis should consider in the differential diagnosis of DHS.

\section{Mitochondrial myopathy}

Mitochondrial myopathy should be part of the differential diagnosis of DHS, particularly when more common causes are excluded. DHS was the presenting manifestation of mitochondrial myopathy in two cases. ${ }^{54}$ Detailed neurological work-up were performed to rule out common causes. The work up revealed elevated serum lactate and mitochondrial proliferation in muscle biopsy. ${ }^{54}$ In another report, DHS was reported as result of possible multi-organ mitochondriopathy, manifesting as Parkinsonism, tetraspasticity, dilative car- 
diomyopathy, short stature, osteoporosis, and myopathy. In this case, neurological examination showed prominent neck extensor weakness, weakness of the shoulder gridle, cogwheel rigidity, and tetraspacticity. The further tests showed elevated serum lactate, myogenic findings in needle EMG, and myogenic abnormalities with weak staining of cytochrome oxidase in muscle biopsy. No improvement of the clinical symptoms with anti-Parkinson medications. Based on those findings, mitochondriopathy with multi-organ involvement was suspected. 55

\section{Other myopathies}

DHS can be caused by several different types of primary or secondary myopathies. It is difficult to summarize all those reports in this article. In this section, we only provide the name of myopathies and its related references. It is paramount important to recognize the causes of secondary myopathies that may be a treatable cause of DHS. Other reasons of secondary myopathies include Cushing syndrome, 56 carnitine deficiency, ${ }^{77-59}$ hypothyroidism, ${ }^{60-62}$ hypokalemia, ${ }^{63-}$ 65 hyperparathyroidism, $66-68$ and post-radiation neck extensor myopathy. ${ }^{69-72}$

\section{Conclusions}

DHS is relatively rare condition that may occur as a result of several neurological, neuromuscular, muscular, and other causes. It is important to differentiate NM and non-NM causes to offer best treatment options to patients. Detailed history taking, physical examinations are key to make an accurate diagnosis. EDx studies, MRI imaging, and paraspinal muscle biopsy can provide valuable information in selected cases. We aim to increase awareness of recognition the entity of dropped head syndrome and factors that may predict response to immunomodulating therapy in dropped head syndrome.

\section{References}

1. Lange DJ, Fetell M, Lovelace RE, et al. The floppy head syndrome. Ann Neurol 1986;20:133.

2. Jokelainen M. Amyotrophic lateral sclerosis in Finland. II: Clinical characteristics. Acta Neurol Scand 1977;56:194204.

3. Gourie-Devi M, Nalini A, Sandhya S. Early or late appearance of "dropped head syndrome" in amyotrophic lateral sclerosis. J Neurol Neurosurg
Psychiatry 2003;74:683-6.

4. Askmark H, Eeg-Olofsson K, Johansson A, et al. Parkinsonism and neck extensor myopathy: a new syndrome or coincidental findings? Arch Neurol 2001;58:232-7.

5. Diederich NJ, Goebel HH, Dooms G, et al. Camptocormia associated with focal myositis in multiple-system atrophy. Mov Disord 2006;21:390-4.

6. Mezaki T. [Clinical characteristics and treatment of dystonia]. Rinsho Shinkeigaku 2011;51:465-70.

7. Fast A, Thomas MA. The "baseball cap orthosis": a simple solution for dropped head syndrome. Am J Phys Med Rehabil 2008;87:71-3.

8. Kawaguchi A, Miyamoto K, Sakaguchi $\mathrm{Y}$, et al. Dropped head syndrome associated with cervical spondylotic myelopathy. J Spinal Disord Tech 2004;17:5314.

9. Nakanishi K, Taneda M, Sumii T, et al. Cervical myelopathy caused by dropped head syndrome. Case report and review of the literature. J Neurosurg Spine 2007;6:165-8.

10. Rahimizadeh A, Soufiani HF, Rahimizadeh S. Cervical Spondylotic Myelopathy Secondary to Dropped Head Syndrome: Report of a Case and Review of the Literature. Case Rep Orthop 2016;2016:5247102.

11. Hoffman D, Gutmann L. The dropped head syndrome with chronic inflammatory demyelinating polyneuropathy. Muscle Nerve 1994;17:808-10.

12. Petiot P, Vial C, de Saint Victor JF, et al. [Dropped head syndrome: diagnostic discussion apropos of 3 cases]. Rev Neurol (Paris) 1997;153:251-5.

13. D'Amelio M, Di Benedetto N, Ragonese P, et al. Dropped head as an unusual presenting sign of myasthenia gravis. Neurol Sci 2007;28:104-6.

14. Spengos K, Vassilopoulou S, Papadimas G, et al. Dropped head syndrome as prominent clinical feature in MuSK-positive Myasthenia Gravis with thymus hyperplasia. Neuromuscul Disord 2008;18:175-7.

15. Ueda T, Kanda F, Kobessho H, et al. "Dropped head syndrome" caused by Lambert-Eaton myasthenic syndrome. Muscle Nerve 2009;40:134-6.

16. Goh KJ, Wong KT, Tan CT. Myopathic dropped head syndrome: a syndrome of mixed aetiology. J Clin Neurosci 2000;7:334-6.

17. Garcin B, Lenglet T, Dubourg O, et al. Dropped head syndrome as a presenting sign of scleromyositis. J Neurol Sci 2010;292:101-3.

18. Fernandez-Serna M, Arboleya L,
Alonso S, et al. Dropped head syndrome in a patient with scleromyositis. J Clin Rheumatol 2013;19:32-4.

19. Katz JS, Wolfe GI, Burns DK, et al. Isolated neck extensor myopathy: a common cause of dropped head syndrome. Neurology 1996;46:917-21.

20. Maruyama T, Hanyu N, Maruyama K, et al. [Clinical and pathological studies on two patients with adult-onset nemaline myopathy]. Rinsho Shinkeigaku 1990;30:738-44.

21. Katirji B, Hachwi R, Al-Shekhlee A, et al. Isolated dropped head due to adultonset nemaline myopathy treated by posterior fusion. Neurology 2005;65:1504-5.

22. Erro M, Echavarri C, Tunon T. [Dropped head syndrome secondary to nemalinic myopathy]. Neurologia 2006;21:376-77.

23. Pasqualin LM, Reed UC, Costa TV, et al. Congenital muscular dystrophy with dropped head linked to the LMNA gene in a Brazilian cohort. Pediatr Neurol 2014;50:400-6.

24. Liewluck T, Milone M, Mauermann ML, et al. A novel VCP mutation underlies scapuloperoneal muscular dystrophy and dropped head syndrome featuring lobulated fibers. Muscle Nerve 2014;50:295-9.

25. Martin AR, Reddy R, Fehlings MG. Dropped head syndrome: diagnosis and management. Evid Based Spine Care J 2011;2:41-7.

26. Hogan KA, Manning EL, Glaser JA. Progressive cervical kyphosis associated with botulinum toxin injection. South Med J 2006;99:888-91.

27. Puruckherr M, Pooyan P, Dube D, et al. The dropped head sign: an unusual presenting feature of myasthenia gravis. Neuromuscul Disord 2004;14:378-9.

28. Gaeta M, Mazziotti S, Toscano A, et al. "Dropped-head" syndrome due to isolated myositis of neck extensor muscles: MRI findings. Skeletal Radiol 2006;35:110-2.

29. Hashimoto K, Miyamoto H, Ikeda T, Akagi M. Radiologic features of dropped head syndrome in the overall sagittal alignment of the spine. Eur Spine J 2018;27:467-74.

30. Suarez GA, Kelly JJ, Jr. The dropped head syndrome. Neurology 1992;42:1625-7.

31. Lin HN, Nagaoka M, Hayashi Y, Yonezawa I. [Pathophysiological analysis of dropped head syndrome caused by various diagnoses - based on surface EMG findings and responses to physiotherapy]. Rinsho Shinkeigaku 2013;53:430-8. 
32. Morino K, Kita Y, Yoneda Y, et al. [Dropped head plus syndrome; a case report]. Rinsho Shinkeigaku. 2003;43:556-9.

33. Oerlemans WG, de Visser M. Dropped head syndrome and bent spine syndrome: two separate clinical entities or different manifestations of axial myopathy? J Neurol Neurosurg Psychiatry 1998;65:258-9.

34. Uemura M, Kosaka T, Shimohata T, et al. Dropped head syndrome in amyotrophic lateral sclerosis. Amyotroph Lateral Scler Frontotemporal Degener 2013;14:232-3.

35. Sih M, Soliven B, Mathenia N, et al. Head-drop: A frequent feature of lateonset myasthenia gravis. Muscle Nerve 2017;56:441-4.

36. Rodolico C, Messina S, Toscano A, et al. Axial myopathy in myasthenia: a misleading cause of dropped head. Muscle Nerve 2004;29:329-30.

37. Swash M. Dropped-head and bent-spine syndromes; axial myopathies? Lancet 1998;352:758.

38. Schranz C, Meinck HM. [Primary neurogenic and myogenic disorders of posture]. Orthopade 2004;33:583-90.

39. Racz A, Giede-Jeppe A, Schramm A, et al. Lambert-Eaton myasthenic syndrome presenting with a "dropped head syndrome" and associated with antibodies against $\mathrm{N}$-type calcium channels. Neurol Sci 2013;34:1253-4.

40. Haegele-Link S, Bohl J, Karajanev P, Urban PP. Chronic inflammatory demyelinating polyradiculoneuropathy (CIDP) as rare differential diagnosis of a dropped head syndrome. Klin Neurophysiol 2006;37:A76.

41. de Oliveira CM, Fussiger H, Winckler PB, Saute JAM. Dropped head syndrome as a manifestation of CharcotMarie-Tooth disease type 4C. Neuromuscul Disord. 2018.

42. Finsterer J, Frank M, Krexner E. Steroid-responsive dropped-head-syndrome due to polymyositis. Joint Bone Spine 2010;77:485-6.

43. Muppidi S, Saperstein DS, Shaibani A, et al. Isolated neck extensor myopathy: is it responsive to immunotherapy? J Clin Neuromuscul Dis 2010;12:26-9.

44. Mirchandani A, Chen AS. Isolated Neck Extensor Myopathy: A Case Presentation of Rapid Onset and Spontaneous Recovery. PM R 2018;10:552-4.

45. Larsen H, Bogaard PW, Oppel L. A case of isolated neck extensor myopathy responding favorably to immunotherapy. J Clin Neuromuscul Dis 2013;15:73-6.

46. Ichikawa Y, Yamada H, Motoyoshi Y, et al. Abnormal head drooping in facioscapulohumeral muscular dystrophy. Rinsho Shinkeigaku 1996;36:5036.

47. Kazakov VM, Bogorodinsky DK, Znoyko ZV, Skorometz AA. The facioscapulo-limb (or the facioscapulohumeral) type of muscular dystrophy. Clinical and genetic study of 200 cases. Eur Neurol 1974;11:236-60.

48. Barbagallo G, Arabia G, Valentino P, et al. Anti-GAD-associated inflammatory myopathy presenting with dropped head syndrome. Neurol Sci 2015;36:1517-9.

49. Lomen-Hoerth C, Simmons ML, Dearmond SJ, Layzer RB. Adult-onset nemaline myopathy: Another cause of dropped head. Muscle Nerve 1999;22:1146-50.

50. Hanisch F, Schneider I, Muller T, et al. [Treatability of sporadic late onset nemaline myopathy]. Nervenarzt 2013;84:955-61.

51. Mizuno Y, Mori-Yoshimura M, Okamoto T, et al. Two cases of sporadic late onset nemaline myopathy effectively treated with immunotherapy. Rinsho Shinkeigaku 2016;56:605-11.

52. Chuquilin M, Al-Lozi M. Primary amyloidosis presenting as "dropped head syndrome". Muscle Nerve 2011;43:905-9.

53. Laurent C, Aouizerate J, Hourdille A, et al. Dropped head syndrome with proximal myopathy revealing AL amyloidosis. Joint Bone Spine 2018;85:779-81.

54. Rahim F, Gupta D, Bertorini TE, Ledoux MS. Dropped head presentation of mitochondrial myopathy. J Clin Neuromuscul Dis 2003;5:108-14.

55. Finsterer J. Dropped head syndrome in mitochondriopathy. Eur Spine J 2004;13:652-6.

56. Rose MR, Levin KH, Griggs RC. The dropped head plus syndrome: quantitation of response to corticosteroids. Muscle Nerve 1999;22:115-8.

57. Karpati G, Carpenter S, Engel AG, et al. The syndrome of systemic carnitine deficiency. Clinical, morphologic, biochemical, and pathophysiologic features. Neurology 1975;25:16-24.

58. Liao JP, Waclawik AJ, Lotz BP, e al. Myopathic dropped head syndrome: an expanding clinicopathological spec- trum. Am J Phys Med Rehabil 2007;86:970-6.

59. Vengalil S, Preethish-Kumar V, Polavarapu K, et al. Fatty acid oxidation defects presenting as primary myopathy and prominent dropped head syndrome. Neuromuscul Disord 2017;27:986-96.

60. Kendall-Taylor P, Turnbull DM. Endocrine myopathies. Br Med J (Clin Res Ed) 1983;287:705-8.

61. Askmark H, Olsson Y, Rossitti S. Treatable dropped head syndrome in hypothyroidism. Neurology 2000;55:896-7.

62. Furutani R, Ishihara K, Miyazawa Y, et al. [A case of hypothyroidism displaying "dropped head" syndrome]. Rinsho Shinkeigaku 2007;47:32-6.

63. Bleck TP. Dropped head syndrome. Neurology 1993;43:846; author reply 846-847.

64. Yoshida S, Takayama Y. Licoriceinduced hypokalemia as a treatable cause of dropped head syndrome. Clin Neurol Neurosurg 2003;105:286-7.

65. Taniguchi K, Okino I, Yamamoto N, et al. [Two cases with dropped head syndrome caused by hypokalemic myopathy]. Rinsho Shinkeigaku 2011;51:1103.

66. Beekman R, Tijssen CC, Visser LH, Schellens RL. Dropped head as the presenting symptom of primary hyperparathyroidism. J Neurol 2002;249: 1738-9.

67. Rymanowski JV, Twydell PT. Treatable dropped head syndrome in hyperparathyroidism. Muscle Nerve. 2009;39:409-10.

68. Ota K, Koseki S, Ikegami K, et al. [Dropped head syndrome as first manifestation of primary hyperparathyroid myopathy]. Rinsho Shinkeigaku 2018;58:193-7.

69. Astudillo L, Hollington L, Game X, et al. Cervical dystonia mimicking dropped-head syndrome after radiotherapy for laryngeal carcinoma. Clin Neurol Neurosurg 2003;106:41-3.

70. Rowin J, Cheng G, Lewis SL, Meriggioli MN. Late appearance of dropped head syndrome after radiotherapy for Hodgkin's disease. Muscle Nerve 2006;34:666-9.

71. Appels C, Goekoop R. Dropped-head syndrome due to high-dose irradiation. J Rheumatol 2009;36:2316.

72. Abdul-Latif AA. Dropped shoulder syndrome: a cause of lower cervical radiculopathy. J Clin Neurol 2011;7:85-9. 